\title{
Une méthode simple et peu coûteuse pour rejeter à l'atmosphère des volumes importants de gaz actifs de courte période
}

\author{
L. QUAGLIA*, J.M. PETERS*, G. DEL FIORE ${ }^{\star \star}$, A. FISSORE ${ }^{\star \star \star}$, \\ J. HANNAY*** \\ (Manuscrit reçu le 4 janvier 1989)
}

\begin{abstract}
RÉSUME
Les concentrations radioactives admissibles lors de rejets de gaz à l'atmosphère doivent respecter les réglementations légales. Dans le cas de courtes périodes, il est possible d'obtenir ce résultat par l'exploitation de la décroissance radioactive en allongeant le temps de transit par passage dans des réservoirs montés en série. L'installation a été étudiée pour un débit d'air de $20 \mathrm{~m}^{3} / \mathrm{h}$ contaminé par le radionucléide ${ }^{15} \mathrm{O}$ de période égale à $124 \mathrm{~s}$. Une soixantaine de fûts de 200 I permettent d'atteindre un facteur d'atténuation de $10^{4}$. Ce facteur devient $3.10^{8}$ pour un débit de $10 \mathrm{~m}^{3} / \mathrm{h}$.
\end{abstract}

\section{ABSTRACT}

The maximum permissible concentration of gazeous radionuclides released in air are stated in legal regulations. For short-lived radionuclides, it is possible to reach these low concentrations by increasing the transit time, before release, by passage through barrels connected in series. The set-up was assessed at a flow rate of $20 \mathrm{~m}^{3} / \mathrm{h}$ of air contaminated with the $124 \mathrm{~s}$ oxygen 15 . An attenuation factor of $10^{4}$ is obtained with sixty 200 । barrels. This factor is $3.10^{8}$ at a flow rate of $10 \mathrm{~m}^{3} / \mathrm{h}$.

\section{INTRODUCTION}

Les cyclotrons médicaux associés à des tomographes à émission de positons se multiplient $[1,6]$. Des gaz tels que $\left[{ }^{15} \mathrm{O}\right] \mathrm{O}^{2}$ et $\left[{ }^{15} \mathrm{O}\right] \mathrm{CO}^{2}$ sont utilisés en routine $[4,7,8,9,11,12]$. Un examen neurologique réalisé par

* Centre de recherches du Cyclotron, Université de Liège, B 4000 Liège, Belgique.

** Institut provincial E. Malvoz, B 4000 Liège, Belgique.

*** Laboratoire de thermodynamique, Université de Liège, B 4000 Liège, Belgique. 
tomographie à émission de positons selon la méthode "à l'équilibre" nécessite la respiration d'un mélange d'air et de gaz radioactif pendant une durée qui peut atteindre $1 \mathrm{~h}$ [5]. L'étanchéité parfaite du masque respiratoire est difficile à réaliser. De plus, le maintien ferme de celui-ci peut être une source d'inconfort telle que les résultats des mesures sont perturbés. Une solution consiste à entourer la tête du patient par une petite hotte. Dans celle-ci, l'air du local est aspiré avec un débit suffisant, pour assurer une vitesse d'entrée acceptable. Le rejet à l'atmosphère se fait souvent par une cheminée. Pour la Belgique, les concentrations radioactives au point d'émission doivent être inférieures à $1,2.10^{5} \mathrm{~Bq} / \mathrm{m}^{3}$ et à $2.10^{4} \mathrm{~Bq} / \mathrm{m}^{3}$ respectivement pour les radionucléides ${ }^{11} \mathrm{C}$ et ${ }^{18} \mathrm{~F}[10]$. Pour l'isotope ${ }^{15} \mathrm{O}$, nous adoptons, pour valeur limite, le dixième de la valeur admise pour ${ }^{18} \mathrm{~F}$, c'est-à-dire $2.10^{3} \mathrm{~Bq} / \mathrm{m}^{3}$.

Dans notre cas, le débit de gaz radioactif arrivant dans le masque est de $11 \mathrm{ml} / \mathrm{s}$. La concentration radioactive est de $4,47.10^{12} \mathrm{~Bq} / \mathrm{m}^{3}$. II s'agit de valeurs habituellement rencontrées dans d'autres laboratoires [3]. Le débit d'air de ventilation rejeté dans la cheminée est de $3,9.10^{6} \mathrm{ml} / \mathrm{s}$. Le facteur de dilution, égal à $3,55.10^{5}$, est insuffisant et la concentration radioactive doit encore être divisée par $0,6.10^{4}$.

Dans le cas où une dilution atmosphérique est autorisée, la situation est plus favorable [2]. Cependant, des difficultés peuvent se présenter lors de conditions atmosphériques difficiles (turbulences, inversions de température...). La construction d'une cheminée adéquate, peut être coûteuse, particulièrement en milieu urbain.

Le stockage dans des réservoirs tampons à pression atmosphérique conduit à une installation encombrante. Dans le cas d'un stockage sous pression, il convient d'assurer une étanchéité suffisante qui augmente le prix de l'installation.

L'écoulement dans un réservoir unique impose des vitesses de quelques millimètres par seconde. Un écart de température de quelques dixièmes de degrés $\mathrm{K}$ entre les parois et l'air provoque un mouvement de convection naturelle dont la vitesse est supérieure à ces valeurs. II est possible de pallier ce fait en augmentant le diamètre du réservoir et en le remplissant de matière poreuse. Cependant, des circuits préférentiels peuvent s'établir au cours du temps suite à l'obturation des pores par les poussières contenues dans l'air.

Nous avons adopté une solution différente qui consiste à allonger le temps de transit, avant le rejet à la cheminée, par passage dans un grand nombre de petits réservoirs montés en série. L'installation a été calculée pour la période de ${ }^{15} \mathrm{O}(124 \mathrm{~s})$ et pour un débit d'air de $20 \mathrm{~m}^{3} / \mathrm{h}$. La circulation est assurée par un petit compresseur placé au pied de la cheminée. Toute l'installation est en légère dépression ce qui supprime tout risque de fuite. 


\section{MODÉLE}

Considérons $2 \mathrm{n}$ réservoirs montés en série, soit:

D : le débit de hotte $\left(\mathrm{m}^{3} / \mathrm{s}\right)$;

$\checkmark \quad$ : le volume d'un réservoir $\left(\mathrm{m}^{3}\right)$;

$\mathrm{T} \quad$ : la période du radionucléide (s);

$\lambda \quad$ : la constante radioactive $\left(\mathrm{s}^{-1}\right), \lambda=0,693 / \mathrm{T}$;

posons : $\lambda^{\star}=\mathrm{D} / \mathrm{V}+\lambda\left(\mathrm{s}^{-1}\right)$;

t : la variable temps (s);

$\mathrm{A}_{i}(\mathrm{t}) \quad$ : l'activité dans tout réservoir i $(\mathrm{Bq})$;

$Q_{i}(t) \quad$ : le débit d'activité à l'entrée du réservoir i $(i>1)(B q / s)$;

$Q_{1} \quad$ : le débit d'activité à l'entrée du réservoir $1 . Q_{1}$ est considéré comme constant;

$q_{i}(t) \quad$ : le débit d'activité à la sortie du réservoir i $(\mathrm{Bq} / \mathrm{s})$;

Nous faisons l'hypothèse que l'homogénéisation dans le réservoir i est instantanée. Désignons par $\mathrm{C}_{i}(\mathrm{t})\left(\mathrm{Bq} / \mathrm{m}^{3}\right)$ la concentration radioactive dans celui-ci. Nous avons:

$$
C_{i}(t)=A_{i}(t) / V
$$

Compte tenu de l'hypothèse, $\mathrm{C}_{\mathrm{i}}(\mathrm{t})$ est la concentration radioactive qui sort du réservoir i. Elle est égale au débit d'activité qui sort du réservoir i divisé par le débit volumique:

$$
C_{i}(t)=q_{i}(t) / D
$$

Désignons par $\mathrm{C}_{0}$ la concentration radioactive constante à l'entrée du premier réservoir :

$$
\mathrm{C}_{0}=\mathrm{Q}_{1} / \mathrm{D}
$$

Le temps de transit entre deux réservoirs successifs est négligeable devant la période. Le débit d'activité à l'entrée du réservoir i est alors égal au débit d'activité à la sortie du réservoir i-1:

$$
\mathrm{Q}_{\mathrm{i}}(\mathrm{t})=\mathrm{q}_{\mathrm{i}-1}(\mathrm{t})
$$

Si nous désignons par ' les dérivés par rapport au temps, la variation d'activité dans le réservoir $i(i>1)$ est donnée par:

$$
A_{i}^{\prime}(t)=Q_{i}(t)-q_{i}(t)-\lambda A_{i}(t)
$$

En tenant compte de la relation (6):

$$
A_{i}^{\prime}(t)=q_{i-1}(t)-q_{i}(t)-\lambda A_{i}(t)
$$

La relation (4) conduit à:

$$
A_{i}^{\prime}(t)=D C_{i-1}(t)-D C_{i}(t)-\lambda A_{i}(t)
$$


En divisant par $\mathrm{V}$ et en tenant compte de la relation (3) il vient:

$$
\begin{aligned}
C_{i}^{\prime}(t) & =\frac{D}{V} C_{i-1}(t)-\frac{D}{V} C_{i}(t)-\lambda C_{i}(t) \\
& =\frac{D}{V} C_{i-1}(t)-\left(\frac{D}{V}+\lambda\right) C_{i}(t)
\end{aligned}
$$

Compte tenu de la relation (2) nous obtenons l'équation différentielle:

$$
C_{i}^{\prime}(t)=\frac{D}{V} C_{i-1}(t)-\lambda^{*} C_{i}(t) \quad(i>1)
$$

avec $C_{i}(0)=0$.

Pour $\mathrm{i}=1$ l'équation (7) s'écrit:

$$
A_{1}^{\prime}(t)=Q_{1}-q_{1}(t)-\lambda A_{1}(t)
$$

qui devient $C^{\prime}{ }_{1}(t)=\frac{Q_{1}}{V}-\lambda^{*} C_{1}(t)$ avec $C_{1}(0)=0$

La solution est donnée par: $C_{1}(t)=\frac{Q}{V \lambda^{\star}}\left(1-e^{-\lambda \star t}\right)$

En introduisant $C_{1}(t)$ dans l'équation (8) pour $i=2$, nous obtenons $C_{2}(t)$. De proche en proche, nous obtenons $C_{i}(t)$ qui s'écrit:

$$
C_{i}(t)=\frac{Q_{1}}{D}\left(\frac{D}{V \lambda^{*}}\right)^{i} \cdot\left[1-e^{-\lambda^{*} t} \cdot \sum_{j=1} \frac{\left(\lambda^{*} t\right)^{j-1}}{(j-1) !}\right]
$$

La concentration radioactive maximale est obtenue à l'équilibre $(\mathrm{t}=\infty): \mathrm{C}_{\mathrm{i}}(\infty)=\frac{\mathrm{Q}_{1}}{\mathrm{D}} \cdot\left(\frac{\mathrm{D}}{\lambda^{\star} \mathrm{V}}\right)^{\mathrm{i}}$

La relation (5) nous donne : $C_{1}(\infty)=C_{0} \cdot\left(\frac{D}{\lambda^{\star} V}\right)^{\prime}$

Le rapport $C_{0} / C_{i}(\infty)$ donne le facteur de réduction de l'activité volumique $F_{i}$ qui, pour $2 n$ réservoirs, s'écrit:

$$
F_{2 n}=\left(\frac{\lambda^{\star} V}{D}\right)^{2 n}
$$

En tenant compte des relations (1) et (2) ce facteur s'écrit:

$$
F_{2 n}=\left(1+\frac{0,693 V}{D T}\right)^{2 n}
$$


Le nombre de réservoirs est alors donné par : $2 n=\frac{1 n F_{2 n}}{\ln \left(1+\frac{0,693 V}{D T}\right)}$

\section{VÉRIFICATION EXPÉRIMENTALE}

L'exactitude des prévisions du modèle repose sur le respect de l'hypothèse d'une homogénéisation rapide et il convient d'en faire la vérification expérimentale.

Pour des raisons économiques, nous avons choisi des fûts cylindriques de fabrication courante. Le diamètre et la hauteur de ces fûts valent respectivement $56 \mathrm{~cm}$ et $88 \mathrm{~cm}$, soit un volume $\mathrm{V}$ égal à $217 \mathrm{I}$. Les parois intérieures de ces fûts sont recouvertes d'un film de polyéthylène.

Pour approcher au mieux l'hypothèse, il convient d'injecter le gaz dans le réservoir avec une vitesse suffisante pour que le dard d'entrée pénètre jusqu'au fond et assure un balayage complet sans volume mort et sans court-circuit importants entre l'entrée et la sortie. L'entrée et la sortie des gaz sont diamétralement opposées sur la même base. Des vitesses d'entrée de 5 à $10 \mathrm{~m} / \mathrm{s}$ devraient assurer un balayage parfait. La vérification est réalisée pour un débit de $10 \mathrm{~m}^{3} / \mathrm{h}$. Cette valeur sera justifiée au paragraphe 4 . Les raccordements de la tuyauterie aux fûts sont réalisés avec des éléments de plomberie ordinaires. Le diamètre du trou d'entrée est de $21 \mathrm{~mm}$. La vitesse correspondante est de $8 \mathrm{~m} / \mathrm{s}$.

La vérification expérimentale utilise deux fûts montés en série. Un compresseur aspire l'air ambiant à travers un compteur volumique permettant de mesurer le débit. Une vanne permet son réglage. Le gaz actif, en l'occurence $\left[{ }^{15} \mathrm{O}\right] \mathrm{O}^{2}$ est amené à l'entrée du compteur. Le mélange gazeux traverse les deux fûts.

Deux sondes $\mathrm{Nal}(\mathrm{TI})$ de 2" x 2" sont placées: l'une à l'entrée du premier fût, l'autre à la sortie du second. Les sondes sont blindées et collimatées. De plus, elles sont placées dans un local distinct de celui où se trouvent les réservoirs. Le mur de béton entre les deux locaux a une épaisseur de $40 \mathrm{~cm}$. L'oxygène 15 est un émetteur $\beta^{+}$pur. L'annihilation a essentiellement lieu dans les parois du tube passant devant les collimateurs. La même géométrie de détection est réalisée pour les deux sondes. Un seuil en énergie est placé au pied du pic photoélectrique pour minimiser l'influence du rayonnement diffusé présent dans le local.

Deux vannes à trois voies permettent de conduire le mélange gazeux d'une sonde à l'autre sans passer par les fûts. Dans ce cas, le temps de transit entre les deux sondes est mesuré par le front d'arrivée de l'activité. Cette disposition permet d'intercalibrer les deux sondes. Le facteur de réduction de l'activité volumique s'obtient en divisant le nombre de coups reçu sur la sonde d'entrée par celui reçu sur celle de sortie. Les taux de 
comptage dans le pic photoélectrique sont de quelques milliers de coups par seconde. Ceci assure une bonne précision statistique sans dépassement de la zone de linéarité du dispositif de mesure.

L'expérience conduit à un facteur de réduction de l'activité volumique égal à 2,02. La valeur théorique calculée par la relation (10) vaut 2,06. Ceci signifie qu'une vitesse d'injection de $8 \mathrm{~m} / \mathrm{s}$ assure un balayage répondant à l'hypothèse du modèle.

\section{INSTALLATION}

Nous disposons d'un local qui permet de mettre en place 62 fûts $(2 n=62)$. Compte tenu du type de fût adopté, il convient d'éviter une perte de charge trop importante qui conduirait à une flexion prohibitive des parois latérales des réservoirs placés le plus en aval de la ligne.

A cette fin, l'installation comporte deux ensembles montés en parallèle. Chaque groupe comprend 31 fûts en série. La relation (10) donnant le facteur de réduction de l'activité volumique $F_{2 n}$ devient:

$$
F_{n}=\left(1+\frac{0,693 V}{\frac{D}{2} \cdot T}\right)^{n}
$$

En remplaçant $\mathrm{V}$ et $\mathrm{n}$ par leur valeur et en considérant la période de $124 \mathrm{~s}$ de l'oxygène 15 , il vient:

$$
F_{n}=\left(1+\frac{0,00243}{D}\right)^{31}
$$

Le modèle suppose que la pression est constante dans tous les réservoirs. En réalité, avec les pertes de charge en ligne, et entre les résérvoirs, le débit volumique des effluents augmente tout au long du parcours. Pour une perte de charge calculée de 0,2 bar, le facteur de réduction de l'activité volumique est divisé par 4 . Pour plus de sécurité, nous considérons que ce facteur est divisé par 10 et donné par:

$$
F_{n}=0,1 .\left(1+\frac{0,00243}{D}\right)^{31} \quad D \text { en } \frac{m^{3}}{s}
$$

Le choix du débit résulte d'un compromis entre deux exigences contradictoires: un débit trop important diminue la valeur de $F_{n}$, par contre un débit trop faible peut conduire à l'entrée de la hotte à une vitesse d'aspiration d'air insuffisante.

Pour un débit de $0,00556 \mathrm{~m}^{3} / \mathrm{s}\left(20 \mathrm{~m}^{3} / \mathrm{h}\right)$ le facteur de réduction $F_{\mathrm{n}}$ vaut $0,75.10^{4}$. Par une construction soignée, l'ouverture d'entrée de la hotte peut se limiter à $0,02 \mathrm{~m}^{2}$. Ceci conduit à une vitesse d'entrée acceptable de 0,28 m/s. 
Les fûts, installés dans le sous-sol du bâtiment, sont disposés horizontalement et empilés en trois couches correspondant à une hauteur totale de 1,6 m. La longueur de l'ensemble est de $12 \mathrm{~m}$. L'encombrement en largeur, des fûts et de leurs tuyaux de raccordement, est de $1,5 \mathrm{~m}$.

\section{CONCLUSIONS}

L'installation décrite est en fonctionnement depuis plusieurs mois et donne entière satisfaction. Le point le plus délicat a été rencontré dans la construction de la hotte entourant la tête du patient. Celle-ci doit être suffisamment étanche pour interdire tout reflux lors de la respiration. Par ailleurs, elle doit permettre le mouvement du lit du tomographe et celui du support des anneaux de détection.

De plus, le positionnement de la tête doit rester aisé. Ces contraintes sont rencontrées par la réalisation d'une tente en plastique souple translucide fixée au tomographe et rabattue sur la poitrine du patient. Des manchons dégagent les bras et permettent les prises sanguines. Un débit de 10 $\mathrm{m}^{3} / \mathrm{h}$ conduit à un facteur de réduction de l'activité volumique égal à $3.10^{8}$. Cependant, un tel débit ne permet pas d'éviter tout reflux et la dilution à la cheminée reste nécessaire pour atteindre le facteur de réduction imposé. Dans le cas où cette dilution serait insuffisante, voire inexistante, et si on ne peut doubler le nombre de fûts, il convient d'utiliser un masque respiratoire mais sans prétendre réaliser une étanchéité parfaite. Le masque reprend la plus grosse partie de l'activité et atténue les à-coups de la respiration. La tente peut alors fonctionner avec un débit de $10 \mathrm{~m}^{3} / \mathrm{h}$. L'installation est peu coûteuse, sans risque de fuites et d'un emploi facile. Pour autant que l'on dispose de la place nécessaire, elle offre une alternative originale aux rejets de gaz actifs de courte période.

\section{RÉFÉRENCES}

[1] ACPN/SNM. - Task force on clinical PET. J. Nucl. Med., 1988, 29, 1136-1143.

[2] BULTYNCK H., FIEW G. - Bepaling van de bromtermen voor lozingen in de atmosfeer en in de waterlopen uitgande van de praktische kennis van de diffusieve kapaciteit van het milieu. In: Comptes rendus du Colloque international L'énergie nucléaire et l'environnement organisé par l'Association des ingénieurs Montéfiore, Liège, janvier 1973.

[3] CLARK J.C., BUCKINGHAM P.D. - Short-lived radioactive gases for clinical use. London: Butterworths, 1975.

[4] CORREIA J.A., ALPERT N.M., BUXTON R.B., ACKERMAN R.H. - Analysis of some errors in the measurement of oxygen extraction and oxygen consumption by the equilibrium inhalation method. J. Cereb. Blood Flow Metab., 1985, 5, 591-599.

[5] FRACKOWIAK R.S.J., LENZI G.L., JONES T., HEATHER J.D. - Quantitative measurement of regional cerebral blood flow and oxygen metabolism in man using ${ }^{15} \mathrm{O}$ and positron emission tomography: theory, procedure, and normal values. J. Comput. Assist. Tomogr., 1980, 4 (6), 727-736. 
[6] HARBY K. - Clinical PET : is it time to take the plunge? J. Nucl. Med., 1988, 29, $1751-1757$.

[7] KOEPPE R.A., HOLDEN J.E., IP W.R. - Performance comparison of parameter estimation techniques for the quantitation of local cerebral blood flow by dynamic positron computed tomography. J. Cereb. Blood Flow Metab., 1985, 5, 224-234.

[8] LAMMERTSMA A.A., CORREIA J.A., JONES T. - Stability of arterial concentrations during continuous inhalation of $\mathrm{C}^{15} \mathrm{O}_{2}$ and ${ }^{15} \mathrm{O}_{2}$ and the effects on computed values of CBF and $\mathrm{CMRO}_{2}$. J. Cereb. Blood Flow Metab., 1988, 8, 411-417.

[9] LEBRUN-GRANDIE P., BARON J.C., SOUSSALINE F., LOC'H C., SASTRE J. BOUSSER M.G. - Coupling between cerebral blood flow and oxygen utilization in the normal human brain. Arch. Neurol., 1983, 40, 230-236.

[10] Arrêté Rcyal du 11 février 1987. Moniteur Belge.

[11] PANTANO P., BARON J.C., CROUZEL C., COLLARD P., SIROU P., SAMSON Y. The ${ }^{15} \mathrm{O}$ continuous-inhalation method: correction for intravascular signal using $\mathrm{C}^{15}$ O. Eur. J. Nucl. Med., 1985, 10, 387-391.

[12] SENDA M., BUXTON R.B., ALPERT N.M., CORREIA J.A., MACKAY B.C., WEISE S.B., ACKERMAN R.H. - The ${ }^{15}$ O steady-state method: correction for variation in arterial concentration. J. Cereb. Blood Flow Metab., 1988, 8, 681-690. 